\title{
Biweekly cetuximab and irinotecan as second-line therapy in patients with gastro-esophageal cancer previously treated with platinum
}

\author{
Katrine R. Schoennemann · Jon K. Bjerregaard · Tine P. Hansen · Karin De Stricker • \\ Morten F. Gjerstorff · Helle A. Jensen $\cdot$ Lene W. Vestermark $\cdot$ Per Pfeiffer
}

Received: 4 October 2010/Accepted: 24 January 2011/Published online: 17 March 2011

(c) The International Gastric Cancer Association and The Japanese Gastric Cancer Association 2011

\begin{abstract}
Background Until recently there has been no proven second-line therapy for patients with advanced gastroesophageal cancer (GEC). Since 2004, Denmark has had a national health program where non-proven therapy can be offered to patients with advanced cancer, after approval by an expert panel appointed by the National Board of Health. This program has accelerated the introduction and implementation of new therapies in Denmark. Inspired by therapy in metastatic colorectal cancer, a combination of cetuximab and irinotecan (Cetiri) was chosen for secondline therapy in GEC patients. We report our experience with Cetiri as second-line therapy in patients with GEC. Methods All patients had histologically confirmed GEC and all patients had progressive disease during or after firstline platinum-containing chemotherapy. The patients received cetuximab $500 \mathrm{mg} / \mathrm{m}^{2}$ on day 1 and irinotecan $180 \mathrm{mg} / \mathrm{m}^{2}$ on day 1 every 2 nd week until progression or unacceptable toxicity. Toxicity was prospectively evaluated
\end{abstract}

K. R. Schoennemann $(\bowtie) \cdot$ J. K. Bjerregaard ·

H. A. Jensen - L. W. Vestermark - P. Pfeiffer

Department of Oncology, Odense University Hospital,

Sdr. Boulevard 29, 5000 Odense C, Denmark

e-mail: k.schoennemann@ouh.regionsyddanmark.dk

T. P. Hansen - K. De Stricker

Department of Clinical Pathology,

Odense University Hospital, Odense C, Denmark

K. R. Schoennemann · J. K. Bjerregaard · P. Pfeiffer Institute of Clinical Research, University of Southern Denmark, Odense C, Denmark

\section{F. Gjerstorff}

Department of Hematology,

Center for Cancer Immune Therapy (CCIT),

Herlev University Hospital, Herlev, Denmark according to the National Cancer Institute Common Terminology Criteria for Adverse Events (NCI-CTCAE) version 3.0.

Results From December 2007 to February 2009, 50 consecutive patients received Cetiri as second-line therapy. Median performance status (PS) was 1. The median number of courses was seven. Seven patients (14\%) had a partial response. Median progression-free survival (PFS) was 3.3 months and overall survival (OS) was 5.5 months; two patients are still alive without progressive disease. Major toxicities were: diarrhea (8\%), fatigue (10\%), neutropenia (16\%), and febrile neutropenia (2\%).

Conclusion Cetiri every two weeks is a convenient and well-tolerated second-line regimen in GEC patients. A promising effect was seen in patients with PS 0-1 and in patients who developed a rash.

Keywords Stomach neoplasm - Cetuximab - Irinotecan · KRAS protein $\cdot$ Second-line therapy

\section{Introduction}

Despite a declining incidence of gastro-esophageal cancer (GEC) in developed countries it is still the second most common cause of cancer-related deaths worldwide [1, 2]. For patients with non-resectable disease or recurrent disease, palliative chemotherapy prolongs survival and improves quality of life [3, 4]. Adenocarcinoma of the esophagus, esophagogastric junction, and the stomach is often regarded as a single entity in regard to the efficacy and toxicity of chemotherapy [5]. At present, there are several standards for chemotherapy regimens for first-line therapy, but most often platinum-based therapies are used, with response rates (RRs) between 25 and $45 \%$, and a prolongation of median 
overall survival (OS) to 7-10 months [6, 7]. Patients who progress on first-line therapy have a dismal prognosis, with an expected median survival of a few months. However, many patients still have an excellent performance status (PS), leading to interest in second-line therapy. Until recently, there was no established second-line therapy in GEC patients, but a new small randomized study demonstrated a prolonged OS in favor of irinotecan compared to best supportive care [8]. Irinotecan is a topoisomerase-1 inhibitor with proven activity in GEC patients, both as a single agent and in combination with other modalities, including 5-fluorouracil (FU) and cisplatin [9-11]. Epidermal growth factor receptor (EGFR) is over-expressed in $40-60 \%$ of GEC patients. Cetuximab is a monoclonal antibody against the EGFR and has shown activity in metastatic colorectal cancer (mCRC) where response to therapy is strongly correlated with the mutational status of KRAS. The combination of cetuximab and irinotecan is active in irinotecan-refractory mCRC patients, with a favorable toxicity profile [12]. Usually cetuximab is administered on a weekly schedule, but pharmacokinetic studies in patients with mCRC have demonstrated no major differences between cetuximab $250 \mathrm{mg} / \mathrm{m}^{2}$ weekly versus cetuximab $500 \mathrm{mg} / \mathrm{m}^{2}$ every second week [13-15]. Administration of a simplified regimen that does not compromise efficacy is desirable for both patients and hospital personnel.

Since 2004, Denmark has had a national health program where non-proven therapy can be offered to patients with advanced cancer, after approval by a Second Opinion Panel appointed by the National Board of Health. This program has had a major impact on the management of cancer patients in Denmark and has accelerated the introduction and implementation of new drugs [16]. Inspired by the high incidence of EGFR over-expression in GEC patients and the promising results seen in patients with $\mathrm{mCRC}$, cetuximab and irinotecan (Cetiri) was administered every second week [17] as experimental second-line therapy in GEC patients whose disease had progressed during or after platinum-based chemotherapy.

\section{Patients, materials, and methods}

In this compassionate-use series, 50 consecutive patients were registered and treated in a single experimental cancer unit. Treatment was, in all patients, recommended by the Danish Second Opinion Panel. Before the start of treatment, patients were evaluated and informed about the experimental therapy with biweekly Cetiri. All patients selected for treatment had histologically verified, evaluable or non-evaluable, non-resectable adenocarcinoma of the esophagus, esophageal junction, or stomach. All patients had received prior platinum-based chemotherapy and had demonstrated progressive disease during or after previous treatment. Standard adequate laboratory tests, including hematology and renal and hepatic function tests, were required prior to treatment. Response was evaluated by the investigators according to the Response Evaluation Criteria in Solid Tumors (RECIST) 1.0. Patients with non-measurable disease at the start of treatment and without signs of progression after 4 cycles of Cetiri (2 months) were considered to have stable disease (SD).

\section{Treatment}

It was planned that patients were to receive a combination of irinotecan $180 \mathrm{mg} / \mathrm{m}^{2}$ and cetuximab as a $500 \mathrm{mg} / \mathrm{m}^{2}$ infusion on day 1 (Cetiri) every second week [17]. The first course of cetuximab was infused in $120 \mathrm{~min}$ ( $\mathrm{min}$ ) followed $1 \mathrm{~h}$ later by irinotecan. Subsequent courses of cetuximab were infused in only $60 \mathrm{~min}$, immediately followed by irinotecan given in $30 \mathrm{~min}$, resulting in an overall treatment time of $90 \mathrm{~min}$. Therapy was repeated every second week until unacceptable toxicity or disease progression. Patients received premedication with oral prednisolone $100 \mathrm{mg}$ and an antihistamine, e.g., $2 \mathrm{mg}$ clemastine i.v., to minimize the risk of infusion-related reactions associated with cetuximab, as well as antiemetics and oral ondansetron $8 \mathrm{mg} \times 2$ before the irinotecan infusion. In order to prevent irinotecan-associated acute cholinergic syndrome, $0.25 \mathrm{mg}$ atropine was given s.c. or as a slow i.v. infusion. Two patients were offered irinotecan $\left(250 \mathrm{mg} / \mathrm{m}^{2}\right)$ and panitumumab $(9 \mathrm{mg} / \mathrm{kg})$ every three weeks due to their having an exceptionally long transportation time to the hospital.

\section{DNA extraction and KRAS mutation analysis}

KRAS mutation analysis was performed on tissue samples from the first 28 patients treated with Cetiri. The tumor samples were obtained from the primary gastric tumors $(n=23)$ or metastatic tissue $(n=5)$. DNA was extracted from formalin-fixed paraffin-embedded (FFPE) tissue sections, using a MagNA Pure LC DNA Isolation Kit (Tissue) (Roche). Samples were included for the analysis after histological verification of the presence of more than $70 \%$ tumor cells in each tumor sample. KRAS mutation analysis was performed as a real-time polymerase chain reaction (PCR), using a TheraScreen ${ }^{\circledR}$ KRAS mutation kit (DxS, Manchester, England) according to the manufacturer's protocol. The seven most common KRAS point mutations in codons 12 and 13 were detected in the analysis. Each sample was analyzed in duplicate.

Evaluation of toxicity and dose adjustment

Adverse events were prospectively evaluated and recorded before each course according to the National Cancer 
Institute Common Terminology Criteria for Adverse Events (CTCAE) version 3.0. In patients with CTCAE grade 3 or 4 adverse events, the dose of irinotecan was reduced by $25 \%$ in the subsequent treatment cycles. If patients developed grade 3 skin toxicity, the dose of cetuximab was postponed until recovery to grade $\leq 2$; in those with recurrent episodes of grade 3 skin toxicity, the dose of cetuximab was reduced by $20 \%$ in the subsequent treatment cycles.

\section{Statistical considerations}

Progression-free survival (PFS) was calculated as the period from the first infusion of Cetiri to the first observation of radiological or clinical disease progression or death of any cause. OS was calculated as the period from the first infusion of Cetiri until death of any cause. Data were updated in December 2010. Survival analyses were performed using the Kaplan-Meier method. In order to establish prognostic factors for OS, a multivariate model was fitted, using Cox regression, to known and collected clinically relevant prognostic factors. Because of the limited numbers of patients only six parameters were included in the multivariate Cox regression model. Performance status (PS) was divided into three groups; $\mathrm{PS}=0$, versus $\mathrm{PS}=1$ versus $\mathrm{PS}=2$. Time to progression (TTP) under first-line chemotherapy was included as a continuous variable and was defined as the period from the start of first-line chemotherapy to the first date of Cetiri. The number of metastatic sites was divided into two groups; $1-2$ versus $\geq 3$. Skin toxicity was included in the model as any rash versus no rash, and blood samples were dichotomized at the upper normal limit.

\section{Results}

\section{Patient characteristics}

From December 2007 to February 2009, 50 consecutive patients were treated at our experimental unit. Patient characteristics are listed in Table 1. Median age was 61 years (range 29-76). The majority of patients (40) were men $(80 \%)$. The location of the primary tumor was the esophagus in two patients, gastro-esophageal junction in 32 , and stomach in 16 . Forty-five patients had metastatic disease. At baseline 17 patients had PS 0 (34\%), 27 patients had PS $1(54 \%)$, and six patients had PS 2 (12\%). All patients had received prior platinum-based combination chemotherapy.

\section{Toxicity}

All patients were evaluable for toxicity. In general, the treatment was well tolerated; the worst CTCAE toxicities
Table 1 Baseline characteristics

\begin{tabular}{|c|c|}
\hline Number of patients & 50 \\
\hline Age, years (median) range & 61 (range 29-76) \\
\hline Male/Female & $40 / 10$ \\
\hline \multicolumn{2}{|l|}{ PS } \\
\hline Median & 1 \\
\hline 0 & $17(34 \%)$ \\
\hline 1 & $27(54 \%)$ \\
\hline 2 & $6(12 \%)$ \\
\hline \multicolumn{2}{|l|}{ Primary tumor site, $n(\%)$} \\
\hline Esophagus & $2(4)$ \\
\hline Gastro-esophageal junction & $32(64)$ \\
\hline Stomach & $16(32)$ \\
\hline \multicolumn{2}{|l|}{ Stage, $n(\%)$} \\
\hline Locally advanced & $5(10)$ \\
\hline Metastatic & $45(90)$ \\
\hline \multicolumn{2}{|l|}{ Number of metastatic sites, $n(\%)$} \\
\hline $1-2$ & $25(50)$ \\
\hline$\geq 3$ & $25(50)$ \\
\hline Median TTP under first-line CT (range) & $\begin{array}{l}4.5 \text { months }(1.5-13) \\
\text { months }\end{array}$ \\
\hline
\end{tabular}

$P S$ performance status, TTP time to progression, $C T$ chemotherapy

are listed in Table 2) Non-hematological toxicities were mainly grade $1-2$; fatigue $(72 \%)$, diarrhea $(48 \%)$, nausea $(70 \%)$, vomiting $(30 \%)$, and skin toxicity $(65 \%)$. Only four patients $(8 \%)$ had diarrhea grade 3 . Hematological toxicities of grade 3-4 were neutropenia (16\%) and febrile neutropenia (2\%). Two patients died before receiving the second course of therapy, one patient due to intestinal volvulus and one patient due to pulmonary embolism.

\section{KRAS mutation analysis}

Mutation analysis of KRAS codons 12 and 13 was retrospectively planned in all patients. However, in eight of the first 28 patients it was not possible to conduct the KRAS mutation analysis because of very sparse tumor tissue. Among the 20 patients analyzed, only two tumors $(10 \%)$ had a KRAS mutation, one in p.Gly12Asp and one in p.Gly12Cys. Eighteen tumors were KRAS wild-type. The two patients with the KRAS mutation received 1 and 28 courses of Cetiri, respectively. The patient receiving only one cycle was hospitalized shortly after the first treatment with intestinal volvulus and died following this. The other patient, who received 28 courses, obtained a partial response (PR) during treatment and had an $\mathrm{R} 0$ resection. Because of the low incidence of KRAS mutations among the analyzed patients, corresponding to results from the literature [18], we decided not to perform KRAS analysis in the remaining patients. 
Table 2 Worst adverse events per patient

\begin{tabular}{llll}
\hline & $\begin{array}{l}\text { Grade } 1+2 \\
n(\%)\end{array}$ & $\begin{array}{l}\text { Grade 3-4 } \\
n(\%)\end{array}$ & $\begin{array}{l}\text { Grade 5 } \\
n(\%)\end{array}$ \\
\hline $\begin{array}{llll}\text { Hematological toxicity } \\
\text { Leucopenia }\end{array}$ & $4(8)$ & $4(8)$ & 0 \\
Neutropenia & $4(8)$ & $8(16)$ & 0 \\
Thrombocytopenia & 0 & 0 & 0 \\
Febrile neutropenia & - & $1(2)$ & 0 \\
Non-hematological toxicity & & & \\
Diarrhea & $24(48)$ & $4(8)$ & 0 \\
Nausea & $35(70)$ & 0 & 0 \\
Vomiting & $15(30)$ & $1(2)$ & 0 \\
Fatigue & $36(72)$ & $5(10)$ & 0 \\
Skin toxicity & $31(65)$ & $1(2)$ & 0 \\
Intestinal volvulus & 0 & 0 & $1(2)$ \\
Pulmonary embolism & 0 & 0 & $1(2)$ \\
\hline
\end{tabular}

Table 3 Efficacy of Cetiri as second-line therapy in GEC patients

\begin{tabular}{ll}
\hline & $n(\%)$ \\
\hline Evaluable disease & $48(96)$ \\
Non-evaluable disease & $2(4)$ \\
Number of courses & 478 \\
$\quad$ Total & $7(1-46)$ \\
Median & \\
Response rate & $7(14)$ \\
PR & $28(56)$ \\
SD & 3.3 months (CI 1.9-4.4) \\
Median PFS & 5.5 months (CI 3.6-7.3) \\
Median OS &
\end{tabular}

Cetiri cetuximab and irinotecan, GEC gastro-esophageal cancer, $P R$ partial response, $S D$ stable disease, $P F S$ progression-free survival, $O S$ overall survival, $C I$ confidence interval

\section{Efficacy}

In total, 478 treatment cycles were administered to 50 patients, with a median of seven cycles per patient (range 1-46) (Table 3). Forty-five patients were evaluable for response because treatment was stopped in five patients before the planned response evaluation after four courses. Reasons for early cessation were toxicity in two patients, death in two other patients (intestinal volvulus and pulmonary embolism), and one due to patient's request. The overall RR was $14 \%$. Ten patients had progressive disease either before or at the time of their first evaluation. The median PFS was 3.3 months and OS was 5.5 months (Fig. 1). After a median follow-up of 29 months, one patient is continuing treatment without signs of progressive disease (PD) and two patients are still alive.

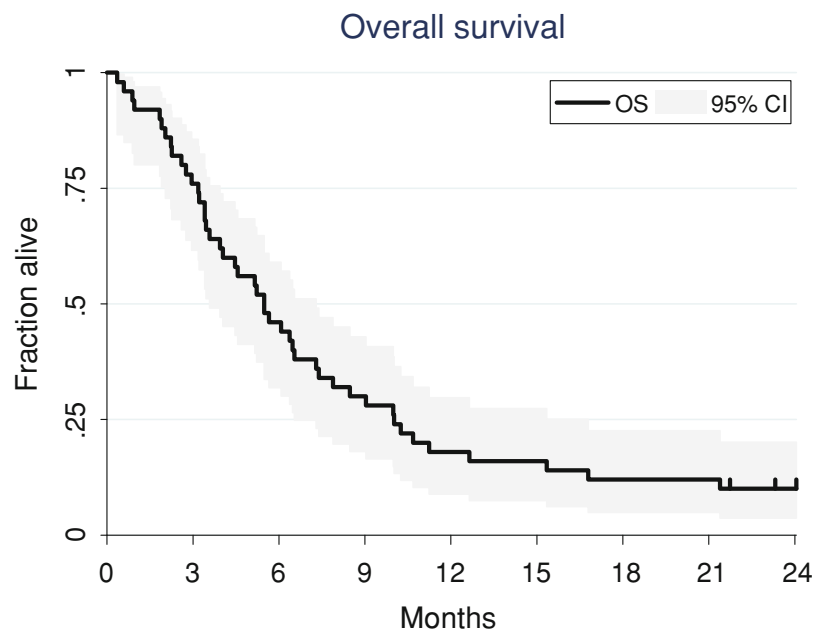

Number at risk

$\begin{array}{llllllllll}\text { All } & 50 & 38 & 23 & 15 & 9 & 8 & 6 & 6 & 3\end{array}$

Progression free survival

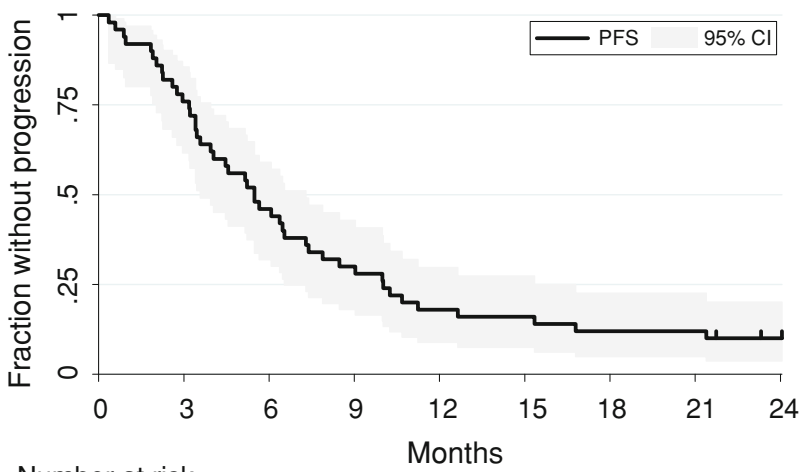

Number at risk

$\begin{array}{llllllllll}\text { All } & 50 & 38 & 23 & 15 & 9 & 8 & 6 & 6 & 3\end{array}$

Fig. 1 Overall survival $(O S)$ and progression-free survival (PFS). Kaplan-Meier curves of overall survival (median 5.5 months) and time to progression (median 3.3 months). CI Confidence interval

In the multivariate analysis PS $=1$ and PS $=2$ were significantly associated with a short OS. Furthermore, the development of rash was significantly associated with a prolonged OS (Table 4).

\section{Discussion}

After progression to first-line chemotherapy, patients with GEC have a dismal prognosis, with an expected OS of a few months. A subgroup of patients is, however, in a good PS at the time of progression and for these patients there is a need for effective second-line chemotherapy. At present, it is often daily clinical practice to offer second-line therapy to patients in a good PS, and 30-40\% of all GEC patients receive second-line therapy after first-line progression 
Table 4 Results of exploratory Cox-multivariate analysis of known and collected parameters for overall survival

\begin{tabular}{lcl}
\hline Parameter & $\begin{array}{l}\text { Hazard ratio } \\
(\mathrm{HR})\end{array}$ & $\begin{array}{l}\text { 95\% Confidence } \\
\text { interval }(\mathrm{CI})\end{array}$ \\
\hline Performance status 1 & $2.4^{*}$ & $1.12-5.32$ \\
Performance status 2 & $10.3^{*}$ & $2.45-43.64$ \\
$\mathrm{WBC}>10 \times 10^{9} / \mathrm{L}$ & 1.7 & $0.9-3.45$ \\
Platelets $>400 \times 10^{9} / \mathrm{L}$ & 1.2 & $0.44-3.1$ \\
Rash & $0.4^{*}$ & $0.17-0.81$ \\
Number of metastatic sites & 1.6 & $0.77-3.17$ \\
TTP under first-line CT & 1.0 & $0.99-1.0$ \\
\hline
\end{tabular}

$* p<0.05$

[19-21]. Several phase II trials have investigated different drugs and combinations in the second-line setting, and promising agents such as irinotecan, taxanes, and platins have been tested $[10,11,22,23]$. So far, no standard regimen has been established as second-line treatment. The need to find prognostic and predictive factors indicating who will benefit from both first- and second-line therapy is imperative. Recently analyses have been performed in order to clarify this question. Chau et al. [19] pooled data on 1080 patients from three randomized first-line trials and demonstrated that $\mathrm{PS} \geq 2$, the presence of liver and/or peritoneal metastases, and elevated alkaline phosphatase were prognostic factors correlated with poor outcome in the first-line setting. Subsequent analyses evaluating patients receiving different regimens of chemotherapy in the second-line setting have described $\mathrm{PS} \geq 2$, number of metastatic sites, short TTP under first-line chemotherapy, and different laboratory test results, such as low hemoglobinand elevated WBC or alkaline phosphatase as prognostic factors associated with overall survival [24-27].

Today the use of molecular targeting agents is a new challenge in modern cancer therapy and these agents may have a significant impact on the treatment of GEC patients in the future [28]. EGFR is over-expressed in many GEC patients and has, in some studies, been associated with a poor prognosis, underlining the significant role that the EGFR might have in human GEC biology. Preclinical trials in EGFR over-expressing gastric cancer cell lines and tumor xenografts have suggested an anti-tumor effect of cetuximab as a result of different intracellular and immunological mechanisms [29, 30]. Moreover, one recently described mechanism of resistance to cetuximab, mutation of the KRAS gene, seems to have a very low prevalence of $8 \%$ in GEC cancer $[17,31]$. These findings are concurrent with the results seen in our study, where a KRAS mutation was found in only $10 \%$ of the 20 analyzed tumors.

As first-line therapy, cetuximab in combination with chemotherapy has been evaluated in phase II trials and has obtained promising results. In the Italian FOLCETUX study, Pinto et al. evaluated cetuximab in combination with irinotecan and 5-fluorouracil (5-FU) and recorded an RR of $44 \%(28-61 \%)$ and promising TTP and OS of 8 and 16 months, respectively, indicating potentially better efficacy than that achieved with irinotecan and 5-FU alone [32-34]. Recently other phase II studies have evaluated cetuximab in combination with either docetaxel and cisplatin, or oxaliplatin and 5-FU in the first-line setting and obtained similar results [35-37].

Unfortunately, limited data exist on the efficacy and predictors for the success of cetuximab as salvage therapy in pretreated patients. Stein et al. evaluated weekly irinotecan and cetuximab in 13 heavily pretreated GEC patients. In that study five patients obtained a PR, the overall disease control rate was $62 \%$, and TTP and OS were 2.6 and 3.4 months, respectively [38]. Park et al. performed a retrospective analysis of 32 heavily pretreated patients mainly in PS 2-3. The disease control rate was $28.6 \%$, PFS was 1.7 months, and OS was 3.2 months. In a multivariate analysis PS, skin rash, and metastatic sites were significantly associated with both PFS and OS [39]. Gold et al. [40] evaluated second-line cetuximab as monotherapy in patients with metastatic esophageal adenocarcinoma, but the trial failed to meet its primary endpoint of a median OS of 6.0 months. In a multivariate analysis, our study demonstrated that PS was a very strong prognostic factor. Both PS 1 and PS 2 were significantly associated with short overall survival (PS1: hazard ratio $[\mathrm{HR}]=2.4$; PS2: $\mathrm{HR}=10.3)$ (Table 4). As a consequence we only recommend Cetiri for patients with PS $\leq 1$. Furthermore, we found that the development of rash was significantly associated with OS. It is remarkable that developing a rash had a strong association with prolonged OS $(\mathrm{HR}=0.4)$. Rash, on the basis of treatment with drugs directed against the EGFR, has also been described as a good prognostic factor in studies including patients with mCRC and pancreatic cancer. One could put forward a hypothesis whether GEC patients in the future could be selected for EGFR treatment based on the occurrence of rash after one or two courses of Cetiri. Surprisingly, our series failed to demonstrate that the number of metastatic sites, TTP under first-line chemotherapy, and different laboratory test results were prognostic factors. Cetiri was well tolerated and easily administered in the out-patient setting.

With a disease control rate of $70 \%$ and an RR of $14 \%$ the present consecutive series indicates that patients in a good PS and patients who develop a rash during treatment benefit from irinotecan and cetuximab every second week. However, these findings need validation in prospective trials.

Conflict of interest None of the authors who contributed to this work has any conflicts of interest. 


\section{References}

1. Parkin DM, Bray F, Ferlay J, Pisani P, et al. Global cancer statistics 2002. CA Cancer J Clin. 2005;55:47-108.

2. Klint A, Engholm G, Storm HH, Tryggvadóttir L, Gislum M, Hakulinen T, et al. Trends in survival of patients diagnosed with cancer of the digestive organs in the Nordic countries 1964-2003 followed up to the end of 2006. Acta Oncol. 2010;49:578-607.

3. Pyrhonen S, Kuitunen T, Nyandoto P, Kouri M. Randomized comparison of fluorouracil, epidoxorubicin, and methotrexate (FEMTX) plus supportive care with supportive care alone in patients with non-resectable gastric cancer. Br J Cancer. 1995; 71:587-91.

4. Glimelius B, Ekström K, Hoffman K, Graf W, Sjödén PO, Haglund $\mathrm{U}$, et al. Randomized comparison between chemotherapy plus best supportive care with best supportive care in advanced gastric cancer. Ann Oncol. 1997;8:163-8.

5. Chau I, Norman A. R., Cunningham D., Oates J., Hawkinsv R., Iveson $\mathrm{T}$, et al. Is there a differential chemotherapy effect in patients with locally advanced or metastatic oesophageal $(\mathrm{O})$, oesophago-gastric junction (OGJ) and gastric (G) adenocarcinoma? A pooled analysis of four randomised controlled trials (RTCs). J Clin Oncol 2007 (abstract 75).

6. Cunningham D, Starling N, Rao S, Iveson T, Nicolson M, Coxon F, et al. Capecitabine and oxaliplatin for advanced esophagogastric cancer. N Engl J Med. 2008;358:36-46.

7. Van Cutsem E, Moiseyenko VM, Tjulandin S, Majlis A, Constenla M, Boni C, et al. Phase III study of docetaxel and cisplatin plus fluorouracil compared with cisplatin and fluorouracil as first-line therapy for advanced gastric cancer: a report of the V325 study group. J Clin Oncol. 2006; 24: 4991-7.

8. Thuss-Patience PC, Kretzschmar T, Deist T, Hinke A, Bichev D, Schumacher G, et al. Irinotecan versus best supportive care (BSC) as 2nd-line therapy in gastric cancer. A randomized phase III study of the Arbeitsgemeinschaft Internistische Onkologie (AIO). J Clin Oncol. 2009;27 (suppl) (abstract LBA 2400).

9. Leary A, Assersohn L, Cunningham D, Norman AR, Chong G, Brown G, et al. A phase II trial evaluating capecitabine and irinotecan as second line treatment in patients with oesophagogastric cancer who have progressed on, or within 3 months of platinum-based chemotherapy. Cancer Chemother Pharmacol 2009;64(3):455-62.

10. Chun JH, Kim HK, Lee JS, Choi JY, Lee HG, Yoon SM, et al. Weekly irinotecan in patients with metastatic gastric cancer failing cisplatin-based chemotherapy. Jpn J Clin Oncol. 2004;34:8-13.

11. Ajani JA, Baker J, Pisters PW, Ho L, Feing B, Mansfiels PF. Irinotecan plus cisplatin in advanced gastric or gastro esophageal junction carcinoma. Oncology (Williston Park). 2001;15:52-4.

12. Cunningham D, Humblet $Y$, Siena S, Khayat D, Bleiberg H, Santoro A, et al. Cetuximab monotherapy and cetuximab plus irinotecan in irinotecan- refractory metastatic colorectal cancer. N Engl J Med. 2004;22:337-45.

13. Tabernero J, Pfeiffer P, Cervantes A. Administration of cetuximab every 2 weeks in the treatment of metastatic colorectal cancer: an effective, more convenient alternative to weekly administration? The Oncologist. 2008;13:113-9.

14. Ramanathan RK. Alternative dosing schedules for cetuximab: a role for biweekly administration? Clin Colorectal Cancer. 2008; 7:364-8.

15. Czejka M, Gruenberger B, Kiss A, Farkouh A, Schueller J. Pharmacokinetics of irinotecan in combination with biweekly cetuximab in patients with advanced colorectal cancer. Anticancer Res. 2010;30:2355-60.

16. Pfeiffer P, Nielsen D, Yilmaz M, Iversen A, Vejlø C, Jensen BV, et al. Cetuximab and irinotecan as third line therapy in patients with advanced colorectal cancer after failure to irinotecan, oxaliplatin and 5-fluorouracil. Acta Oncol. 2007;46:697-701.

17. Pfeiffer P, Nielsen D, Bjerregaard J, Qvortrup C, Yilmaz M, Jensen B, et al. Biweekly cetuximab and irinotecan as third-line therapy in patients with advanced colorectal cancer after failure to irinotecan, oxaliplatin and 5-fluorouracil. Ann Oncol. 2008; 19:1141-5

18. Catalogue of somatic mutations in cancer [Internet] [updated 2010 March 8; cited 2010 march 9]. http://www.sanger.ac.uk/ genetics/CGP/cosmic/.

19. Chau I, Norman AR, Cunningham D, Waters JS, Oates J, Ross PJ. Multivariate prognostic factor analysis in locally advanced and metastatic esophagus-gastric cancer-pooled analysis from three multicentre randomized controlled trials using individual patient data. J Clin Oncol. 2004;22:2395-403.

20. Wilson $\mathrm{D}$, Hillert $\mathrm{L}$, Geh $\mathrm{Ji}$, et al. Review of second-line chemotherapy for advanced gastric adenocarcinoma. Clin Oncol. 2005; 17:81-91.

21. Lee J, Lim T, Uhm JE, Park KW, Park SH, Lee SC, et al. Prognostic model to predict survival following first-line chemotherapy in patients with metastatic gastric adenocarcinoma. Ann Oncol. 2007;18:886-91.

22. Sym SJ, Chang HM, Kang HJ, Lee SS, Ryu MH, Lee JL, et al. A phase II study of irinotecan and docetaxel combination chemotherapy for patients with previously treated metastatic or recurrent advanced gastric cancer. Cancer Chemother Pharmacol. 2008;63:1-8.

23. Kim DY, Kim JH, Lee SH, Kim TY, Heo DS, Bang YJ, et al. Phase II study of oxaliplatin, 5-fluorouracil and leucovorin in previously platinum-treated patients with advanced gastric cancer. Ann Oncol. 2003;14:383-7.

24. Catalano V, Graziano F, Santini D, D'Emidio S, Baldelli AM, Rossi D, et al. Second-line chemotherapy for patients with gastric cancer: who may benefit? Br J Cancer. 2008;99:1402-7.

25. Moon TW, Rha SY, Jeung H, Kim C, Hong MH, Chang H, et al. Outcomes of multiple salvage chemotherapy for advanced gastric cancer: implications for clinical practice and trial design. Cancer Chemother Pharmacol. 2010;66:797-805.

26. Hashimoto K, Takashima A, Nagashima K, Okazaki S, Nakajima TE, Kato K, et al. Progression-free survival in first-line chemotherapy is a prognostic factor in second-line chemotherapy in patients with advanced gastric cancer. J Cancer Res Clin Oncol. 2010;136:1059-64.

27. Kanagaval D, Pokataev IA, Fedyanin MY, Tryakin AA, Bazin IS, Narimanov MN, et al. A prognostic model in patients treated for metastatic gastric cancer with second-line chemotherapy. Ann Oncol. 2010;21:1779-85.

28. Becker JC, Muller-Tidow C, Serve H, Domschke W, Pohle T. Role of receptor tyrosine kinases in gastric cancer: new targets for a selective therapy. World J Gastroenterol. 2006;12:3297305.

29. Patel D, Bassi R, Hooper A, Prewett M, Hicklin DJ, Kang X, et al. Anti-epidermal growth factor receptor monoclonal antibody cetuximab inhibits EGFR/HER-2 heterodimerization and activation. Int J Oncol. 2009;34:25-32.

30. Shimura T, Kataoka H, Ogasawara N, Kubota E, Sasaki M, Tanida S. Suppression of proHB-EGF carboxy-terminal fragment nuclear translocation: a new molecular target therapy for gastric cancer. Clin Cancer Res. 2008;14:3956-65.

31. Karapetis CS, Khambata-Ford S, Jonker DJ, O'Callaghan CJ, Tu $\mathrm{D}$, Tebbutt NC, et al. K-ras mutations and benefit from cetuximab in advanced colorectal cancer. N Engl J Med. 2008;359:1757-65.

32. Pinto C, Di Fabio F, Siena S, Cascinu S, Rojas Llimpe FL, Ceccarelli $\mathrm{C}$, et al. Phase II study of cetuximab in combination with FOLFIRI in patients with untreated advanced gastric or 
gastro esophageal junction adenocarcinoma (FOLCETUX study). Ann Oncol. 2007;18:110-7.

33. Dank M, Zaluski J, Barone C, Valvere V, Yalcin S, Peschel C, et al. Randomized phase III study comparing irinotecan combined with 5-fluorouracil and folinic acid to cisplatin combined with 5-fluorouracil in chemotherapy naive patients with advanced adenocarcinoma of the stomach or esophagogastric junction. Ann Oncol. 2008;19:1450-7.

34. Pozzo C, Barone C, Szanto J, Padi E, Peschel C, Bükki J, et al. Irinotecan in combination with 5-fluorouracil and folinic acid or with cisplatin in patients with advanced gastric or esophagealgastric junction adenocarcinoma: results of a randomized phase II study. Ann Oncol. 2004;15:1773-81.

35. Lordick F, Luber B, Lorenzen S, Hegewisch-Becker S, Folprecht G, Wöll E, et al. Cetuximab plus oxaliplatin/leukovorin/5-fluorouracil in first-line metastatic gastric cancer: a phase II study of the Arbeitsgemeinschaft Internististische Onkologie (AIO). Br J Cancer. 2010;102:500-5.

36. Pinto C, Di Fabio F, Siena S, Falcone A, Cascinu S, et al. Phase II study of cetuximab in combination with cisplatin and docetaxel in patients with untreated advanced gastric or gastro-esophageal junction adenocarcinoma (DOCETUX study). $\mathrm{Br} \mathrm{J}$ Cancer. 2009;101:1261-8.

37. Han SW, Oh DY, Im SA, Park SR, Lee KW, Song HS, et al. Phase II study and biomarker analysis of cetuximab combined with modified FOLFOX6 in advanced gastric cancer. Br J Cancer. 2009;100:298-304.

38. Stein A, Al-Batran SE, Arnold D, Peinert S, Siewczynski R, Schmoll HJ. Cetuximab with irinotecan as salvage therapy in heavily pretreated patients with metastatic gastric cancer J Clin Oncol. 2007 (abstract 47).

39. Park SR, Kook M, Choi IJ, Kim CH, Lee JY, Cho SJ, et al. Predictive factors for efficacy of cetuximab plus chemotherapy as salvage therapy in metastatic gastric cancer patients. Cancer Chemother Pharmacol. 2010;65:579-87.

40. Gold J, Goldman B, Iqbal S, Leichman LP, Zhang W, Lenz HJ, et al. Cetuximab as second-line therapy in patients with metastatic esophageal adenocarcinoma. J Thorac Oncol. 2010; 5:1472-6. 